Veysseyre, R., Weigel, D., Phan, T. \& Effantin, J. M. (1984). Acta Cryst. A40, 331-337.

Weigel, D., Phan, T. \& Veysseyre, R. (1987). Acta Cryst. A43, 294-304.

Acta Cryst. (1991). A47, 238-243
Weigel, D. \& Veysseyre, R. (1990). C. R. Acad. Sci. In the press.

Weigel, D., Veysseyre, R. \& Phan, T. (1990). Acta Cryst. A46, 463-466.

\title{
Frequency-Restrained Structure-Factor Refinement. II. Comparison of Methods
}

\author{
By V. Yu. Lunin and E. A. Vernoslova \\ Research Computer Centre, USSR Academy of Sciences, Pushchino, Moscow Region 142292, USSR
}

(Received 21 December 1989; accepted 3 December 1990)

\begin{abstract}
The frequency distribution of electron-density-function values encountered in a protein crystal has a characteristic shape and may be predicted for a protein with unknown spatial structure. It is shown that various methods of refinement of structure-factor phases (frequency-restrained refinement, histogram matching, density modification) may be regarded as various approaches to the same problem of obtaining the electron-density distribution which agrees with the X-ray experimental data and has a prescribed histogram. Test computations illustrate the relative efficiency of the methods analyzed.
\end{abstract}

\section{Introduction}

The method of isomorphous replacement, which is used to solve the phase problem in protein crystallography, fails sometimes to give the desired quality of electron-density-distribution maps. Additional ways of improving maps are needed. One of them is using, or rather trying to use, the knowledge of the mathematical properties of the electron-densitydistribution function, in addition to the data from $\mathrm{X}$-ray experiments. In previous papers (Lunin, 1986, 1988; Lunin, Urzhumtsev \& Skovoroda, 1990; Lunin \& Skovoroda, 1991), we showed that a valuable source of information on a protein can be a histogram corresponding to a finite-resolution image of its distribution function. Analogous approaches were suggested by Luzzati, Mariani \& Delacroix (1988), Harrison (1988) and Zhang \& Main (1990).

Let us recall the main point. Let $\rho(\mathbf{r})$ be a function defined for points $\mathbf{r}$ of a unit cell $V$ and

$$
F(\mathbf{s}) \exp [i \varphi(\mathbf{s})]=\int_{V} \rho(\mathbf{r}) \exp [2 \pi i(\mathbf{s}, \mathbf{r})] \mathrm{d} V_{\mathbf{r}}
$$

be its structure factors.

0108-7673/91/030238-06\$03.00
By the image of $\rho(\mathbf{r})$ at a resolution $d$ we mean the function

$$
\begin{aligned}
\rho_{d}(\mathbf{r})= & (1 /|V|) \sum_{|\mathbf{s}| \leq 1 / d} F(\mathbf{s}) \exp [i \varphi(\mathbf{s})] \\
& \times \exp [-2 \pi i(\mathbf{s}, \mathbf{r})] .
\end{aligned}
$$

We define the cumulative function for the image $\rho_{d}(\mathbf{r})$,

$$
N(t)=(1 /|V|) \operatorname{mes}\left\{\mathbf{r}: \rho_{d}(\mathbf{r}) \leq t\right\},
$$

and its density,

$$
\nu(t)=\frac{\mathrm{d}}{\mathrm{d} t} N(t)=\frac{1}{|V|} \frac{\mathrm{d}}{\mathrm{d} t} \operatorname{mes}\left\{\mathbf{r}: \rho_{d}(\mathbf{r}) \leq t\right\} .
$$

Here $|V|$ is the total volume of the unit cell and mes $\{r: A\}$ is the volume of the part of the cell occupied by points $\mathbf{r}$ satisfying condition $A$. The value $\nu(t) \Delta t$ is the probability that the value $\rho_{d}(\mathbf{r})$ will belong to the interval $(t, t+\Delta t)$ for a random choice of point $\mathbf{r}$ in the cell. It was shown earlier (Podjarny \& Yonath, 1977; Lunin, 1986; Zhang \& Main, 1990) that, if $\rho(\mathbf{r})$ is the function of electron-density distribution in a protein, the graph of the function $\nu(t)$ has a characteristic shape. An approach to calculating the function $\nu(t)$ for proteins with unknown space structure has been suggested (Lunin \& Skovoroda, 1991). [As before, it is called here the histogram of the image $\rho_{d}(\mathbf{r})$.] We denote this a priori defined histogram as $\nu^{a}(t)$ and refer to it as a 'standard' (for a given object and a given resolution).

In practice, the values of phases $\varphi(s)$ and of moduli $F(s)$ used to calculate the image (1) often contain errors. Moreover, part of the phases, and of moduli as well, may be dropped out of calculation. It results in a distorted image $\rho_{d}(\mathbf{r})$.

In this paper we shall show how the a priori knowledge of the histogram $\nu^{a}(t)$ [or, which is equivalent, of the cumulative function $N^{a}(t)$ ] can be used for a more exact determination of the phases and moduli

(C) 1991 International Union of Crystallography 
in the synthesis (1), i.e. for obtaining a more interpretable synthesis. More precisely, it will be shown that various methods of improving the quality of maps [frequency-restrained structure-factor refinement (Lunin, 1988), histogram specification (Harrison, 1988), histogram matching (Zhang \& Main, 1990) and the well known 'density modification' (Sayre, 1952; Hoppe \& Gassmann, 1968; Simonov, 1976; Podjarny, 1987)] can be treated as various approaches to obtain an image $\rho_{d}(\mathbf{r})$ that would agree with the $\mathrm{X}$-ray experimental information and possess the prescribed 'standard' histogram.

For the sake of simplicity, in this paper all moduli $F(\mathbf{s})$ necessary for calculating the synthesis (1) will be assumed to be known exactly and equal to $F^{o}(\mathbf{s})$ and all the phases $\varphi(\mathbf{s})$ to be known approximately and requiring refinement. A more general problem when either some moduli are unknown or some phases are known exactly may be regarded in a similar way.

\section{Frequency-restrained structure-factor refinement (FRR)}

Let $\nu^{a}(t)$ be the standard histogram corresponding to the image $\rho_{d}(\mathbf{r})$ desired. The problem of phase refinement can be formulated as follows:

Problem 1. For known structure-factor moduli $\left\{F^{o}(\mathbf{s})\right\}$, the values of phases $\{\varphi(\mathbf{s})\}$ should be chosen so that the function

$$
\begin{aligned}
\rho_{d}(\mathbf{r})= & (1 /|V|) \sum_{|\mathbf{s}| \leq 1 / d} F^{o}(\mathbf{s}) \exp [i \varphi(\mathbf{s})] \\
& \times \exp [-2 \pi i(\mathbf{s}, \mathbf{r})]
\end{aligned}
$$

has the histogram $\nu^{a}(t)$, i.e. that

$$
\frac{1}{|V|} \frac{\mathrm{d}}{\mathrm{d} t} \operatorname{mes}\left\{\mathbf{r}: \rho_{d}(\mathbf{r}) \leq t\right\}=\nu^{a}(t) .
$$

The most direct approach to solving the problem is

(a) to formulate a criterion of similarity for the standard histogram $\nu^{a}(t)$ and a histogram $\nu^{c}(t)$ corresponding to some trial values of the phases $\left\{\varphi^{c}(\mathbf{s})\right\}$, for example

$$
Q\left[\nu^{c}\right]=\int_{-\infty}^{\infty} \omega(t)\left[\nu^{c}(t)-\nu^{a}(t)\right]^{2} \mathrm{~d} t
$$

[where $\omega(t)$ is a weighting function];

$(b)$ then to find refined phase values by minimizing the criterion $Q\left[\nu^{c}\right]$ somehow. More precisely, let $\left\{\varphi^{c}(s)\right\}$ be trial phase values. We calculate the synthesis

$$
\begin{aligned}
\rho^{c}(\mathbf{r})= & (1 /|V|) \sum_{\mid \mathbf{s} \leq 1 / d} F^{o}(\mathbf{s}) \exp \left[i \varphi^{c}(\mathbf{s})\right] \\
& \times \exp [-2 \pi i(\mathbf{s}, \mathbf{r})]
\end{aligned}
$$

find its histogram

$$
\nu^{c}(t)=\frac{\mathrm{d}}{\mathrm{d} t} N^{c}(t)=\frac{1}{|V|} \frac{\mathrm{d}}{\mathrm{d} t} \operatorname{mes}\left\{\mathbf{r}: \rho^{c}(\mathbf{r}) \leq t\right\}
$$

and determine the value $Q$ of disagreement between the calculated and the standard histogram (4). This value is a function of trial phase values $\left\{\varphi^{c}(s)\right\}$ with $|\mathbf{s}| \leq 1 / d$ and the problem can be reduced to minimization of the function $Q\left[\nu^{c}\right]=Q(\{\varphi(\mathbf{s})\})$. From a viewpoint of computation this problem is similar to those of phase refinement (Sayre, 1972; Lunin, 1985) and can be solved, e.g. by the methods of gradient descent. The examples of application of such an approach have been given by Lunin (1988) and Lunin \& Skovoroda (1991). Practical details of this minimization process will be published elsewhere.

\section{Histogram-matching (HM) method}

\subsection{The transformation restoring histogram}

Let $\rho(\mathbf{r})$ be a function defined for the points of the unit cell and $\nu(t)$ be its histogram (2). Assume this histogram does not coincide with the standard $\nu^{a}(t)$. If we want to transform the function $\rho(\mathbf{r})$ in such a way that the transformed function $\rho^{m}(\mathbf{r})$ would have the prescribed histogram $\nu^{a}(t)$ and retain the structural information that $\rho(\mathbf{r})$ contains we should require that this transformation $\tau: \rho \rightarrow \rho^{m}$ has the following properties:

$$
\begin{gathered}
\frac{1}{|V|} \frac{\mathrm{d}}{\mathrm{d} t} \operatorname{mes}\left\{\mathbf{r}: \rho^{m}(\mathbf{r}) \leq t\right\}=\nu^{a}(t) \\
\text { if } \rho\left(\mathbf{r}_{1}\right)>\rho\left(\mathbf{r}_{2}\right), \text { then } \rho^{m}\left(\mathbf{r}_{1}\right)>\rho^{m}\left(\mathbf{r}_{2}\right)
\end{gathered}
$$

and, vice versa,

$$
\text { if } \rho^{m}\left(\mathbf{r}_{1}\right)>\rho^{m}\left(\mathbf{r}_{2}\right) \text {, then } \rho\left(\mathbf{r}_{1}\right)>\rho\left(\mathbf{r}_{2}\right) \text {. }
$$

The second property means that the transformation $\tau$ does not deform the level surfaces of the function $\rho$. In particular, local maxima of $\rho(\mathbf{r})$ remain those of $\rho^{m}(\mathbf{r})$, though their values change. We call transformation $\tau$ possessing the properties $(a)$ and $(b)$ 'nonlinear stretching restoring histogram $\nu^{a}(t)$ ' (NSRH).

\subsection{Construction of the nonlinear stretching restoring histogram (NSRH)}

It follows from condition (6) that the value of $\rho^{m}(\mathbf{r})$ depends upon the value of $\rho(\mathbf{r})$ only and is independent of the point at which it is regarded. This means the transformation of the prescribed function $\rho(\mathbf{r})$ has the form

$$
\tau: \rho(\mathbf{r}) \rightarrow \rho^{m}(\mathbf{r})=\lambda_{\rho}[\rho(\mathbf{r})],
$$

where $\lambda_{\rho}(t)$ is the function of the real parameter $t$. Here the modifying functions $\lambda_{\rho}(t)$ generally are 
different for various $\rho(\mathbf{r})$. By (6), the function $\lambda_{\rho}(t)$ should increase monotonically in the interval

$$
\rho_{\min }=\min _{\mathbf{r}} \rho(\mathbf{r}) \leq t \leq \max _{\mathbf{r}} \rho(\mathbf{r})=\rho_{\max } .
$$

For the property (5) to occur, it is necessary that $\lambda_{\rho}(t)$ be such that for all $t$

$\frac{1}{|V|} \operatorname{mes}\left\{\mathbf{r}: \lambda_{\rho}[\rho(\mathbf{r})] \leq t\right\}=N^{a}(t)=\int_{-\infty}^{t} \nu^{a}(\tau) \mathrm{d} \tau$.

The function $\lambda_{\rho}(t)$ is monotonic within the interval $\left[\rho_{\min }, \rho_{\max }\right]$, therefore (8) is equivalent to the condition that, for $\rho_{\min } \leq t \leq \rho_{\max }$,

$$
\begin{aligned}
N_{\rho}(t) & =(1 /|V|) \operatorname{mes}\{\mathbf{r}: \rho(\mathbf{r}) \leq t\} \\
& =(1 /|V|) \operatorname{mes}\left\{\mathbf{r}: \lambda_{\rho}[\rho(r)] \leq \lambda_{\rho}(t)\right\} \\
& =N^{a}\left[\lambda_{\rho}(t)\right] .
\end{aligned}
$$

Thus, the function $\lambda_{\rho}(t)$ should satisfy the equation

$$
N^{a}\left[\lambda_{\rho}(t)\right]=N_{\rho}(t) \text { for } \rho_{\min } \leq t \leq \rho_{\max },
$$

where $N^{a}(t)$ is an a priori prescribed cumulative function and $N_{\rho}(t)$ is a cumulative function corresponding to the function $\rho(\mathbf{r})$.

Since for $\rho_{\min }<t<\rho_{\max }$ the function $N^{a}(t)$ increases strictly monotonically (it cannot have constant intervals here because it corresponds to a finite-Fourier series), (9) has a unique solution for each $t \in\left(\rho_{\min }, \rho_{\max }\right)$.

Thus, the image with properties (5)-(6) has the form (7) where the modifying function $\lambda_{\rho}(t)$ is uniquely defined on the interval $\left[\rho_{\min }, \rho_{\max }\right]$ by (9). In other words, the transformation $\tau$ of the function $\rho(r)$ can be done in two stages:

(a) for the function $\rho(\mathbf{r})$ one calculates the cumulative function

$$
N_{\rho}(t)=(1 /|V|) \operatorname{mes}\{\mathbf{r}: \rho(\mathbf{r}) \leq t\}
$$

and defines the modifying function $\lambda_{\rho}(t)$ from

$$
N^{a}\left(\lambda_{\rho}\right)=N_{\rho}(t)
$$

(b) then one performs the modification

$$
\tau[\rho]=\rho^{m}(\mathbf{r})=\lambda_{\rho}[\rho(\mathbf{r})] .
$$

\subsection{Iterative phase refinement}

The solution $\rho^{\text {ex }}(\mathbf{r})$ of problem $1(\S 1)$ cannot be changed by the transformation $\tau$ defined by (10)-(12) [since it inherently has the 'right' histogram, $N_{\rho}(t)=$ $N^{a}(t)$ and $\left.\lambda_{\rho}(t) \equiv t\right]$, therefore

$$
\tau\left[\rho^{\mathrm{ex}}\right]=\rho^{\mathrm{ex}} \text {. }
$$

On the other hand, if the function of form (5) satisfies condition (13), it also satisfies $\lambda_{\rho}(t) \equiv t$ and, consequently, $N^{a}(t)=N_{\rho}(t)$, which means it has the 'right' histogram. This implies equivalence of the requirements that

(a) the functions $\rho(\mathbf{r})$ of form (3) should have the histogram $\nu^{a}(t)$ and

(b) the function $\rho(\mathbf{r})$ of form (3) should possess the property $\tau[\rho]=\rho(\mathbf{r})$ [where NSRH $\tau$ is defined by (10)-(12)], so that problem 1 can be equivalently reformulated as

Problem 1'. For the known moduli $\left\{F^{o}(\mathbf{s})\right\}$ of structure factors the values of phases $\{\varphi(\mathbf{s})\}$ should be chosen so that the function

$$
\begin{aligned}
\rho_{d}(\mathbf{r})= & (1 /|V|) \sum_{|\mathbf{s}| \leq 1 / d} F^{o}(\mathbf{s}) \exp [i \varphi(\mathbf{s})] \\
& \times \exp [-2 \pi i(\mathbf{s}, \mathbf{r})]
\end{aligned}
$$

has the property

$$
\lambda_{\rho}\left[\rho_{d}(\mathbf{r})\right]=\rho_{d}(\mathbf{r}),
$$

where the function $\lambda_{\rho}(t)$ is defined by the condition

$$
N^{a}\left(\lambda_{\rho}\right)=N_{\rho}(t)=(1 /|V|) \text { mes }\left\{\mathbf{r}: \rho_{d}(\mathbf{r}) \leq t\right\} .
$$

Two approaches are possible to solve the problem (Lunin, 1985). We shall concentrate on one of them closely connected with histogram matching and density modification.

Equation (14) is equivalent to the following system of equations for structure factors:

$$
\left\{\begin{aligned}
F^{o}(\mathbf{s}) & =\left|\mathscr{F}_{\mathbf{s}}\{\tau[\rho]\}\right| & & \text { for }|\mathbf{s}| \leq 1 / d \\
0 & =\left|\mathscr{F}_{\mathbf{s}}\{\tau[\rho]\}\right| & & \text { for }|\mathbf{s}|>1 / d \\
\varphi(\mathbf{s}) & =\arg \mathscr{F}_{\mathbf{s}}\{\tau[\rho]\} & & \text { for }|\mathbf{s}| \leq 1 / d,
\end{aligned}\right.
$$

where $\left|\mathscr{F}_{s}\right|$ and $\arg \left(\mathscr{F}_{s}\right)$ are the modulus and the phase of the s-indexed structure factor corresponding to the function $\tau[\rho](\mathbf{r})$.

If we reduce the system to

$$
\varphi(\mathbf{s})=\arg \mathscr{F}_{\mathbf{s}}\{\tau[\rho]\}=\Phi_{\mathbf{s}}(\{\varphi(\mathbf{s})\}), \quad|\mathbf{s}| \leq 1 / d,
$$

we can try to solve it by a simple iteration method. It means that starting from a set of phases $\left\{\varphi_{o}(\mathbf{s})\right\}$ iterations are carried out by the formula

$$
\varphi_{n+1}(\mathbf{s})=\Phi_{\mathbf{s}}\left(\left\{\varphi_{n}(\mathbf{s})\right\}\right), \quad|\mathbf{s}| \leq 1 / d .
$$

The question whether this process converges and, if it does, then to what remains open here.

\subsection{Basic procedure of histogram matching}

The process (15) can be broken down into four steps:

(1) a synthesis $\rho_{n}(r)$ is calculated with exact values of moduli $\left\{F^{o}(\mathbf{s})\right\}$ and approximate values of phases $\left\{\varphi_{n}(\mathbf{s})\right\}$;

(2) its cumulative function

$$
N_{n}(t)=(1 /|V|) \operatorname{mes}\left\{\mathbf{r}: \rho_{n}(\mathbf{r}) \leq t\right\}
$$

is defined to find the NSRH

$$
\lambda_{n}(t) \text { from } N^{a}\left[\lambda_{n}(t)\right]=N_{n}(t) ;
$$


(3) the synthesis is modified as

$$
\rho_{n}^{m}(\mathbf{r})=\lambda_{n}\left[\rho_{n}(\mathbf{r})\right]
$$

(4) the structure factors $F_{n}^{m}(\mathbf{s}) \exp \left[i \varphi_{n}^{m}(\mathbf{s})\right]$, corresponding to the modified function $\rho_{n}^{m}(\mathbf{r})$, are calculated, and their phases are taken as a next approximation, so that

$$
\varphi_{n+1}(\mathbf{s})=\varphi_{n}^{m}(\mathbf{s}) .
$$

This is the principal scheme of the method of histogram matching (Harrison, 1988; Zhang \& Main, 1990). It is a possible way of solving problem $1^{\prime}$ for the solution of the 'phase part' of (14) by a simple iteration method.

\section{Density-modification (DM) method}

We readily see that the procedure described in $\S 2.4$ differs from the 'classical' method of electron-density modification (see, for example, the paper by Podjarny, 1987) but in a more complicated construction of the modifying function $\lambda_{n}(t)$. In the 'classical' scheme the modifying function has the same form in all refinement runs. It may be, for example,

$\lambda_{n}(\rho)=\left\{\begin{array}{l}{\left[3\left(\rho / \rho_{\max }^{(n)}\right)^{2}-2\left(\rho / \rho_{\max }^{(n)}\right)^{3}\right] \quad \text { for } \rho \geq 0} \\ 0 \text { for } \rho<0 .\end{array}\right.$

Here again the modifying function $\lambda_{n}(\rho)$ depends on the transformed function $\rho_{n}(\mathbf{r})$ or, to be more exact, on its maximum value $\rho_{\max }^{(n)}$.

To establish a closer connection between the modifying functions in HM and DM methods, we will consider a limiting case - the construction of a NSRH for an electron-density synthesis calculated with random phases.

Fig. 1(a) shows histograms corresponding to a synthesis constructed with the exact values of structure-factor moduli and phases and to a synthesis constructed with the exact values of moduli and random values of phases. The histograms with inexact phases are between these two extreme situations.

Fig. 1(b) shows the plots of the cumulative function $N^{e}(t)$ corresponding to the exact synthesis and of the function $N^{r}(t)$ corresponding to a synthesis with random phases, and Fig. $1(c)$ is the plot of the modifying function $\lambda(t)$ defined by $N^{r}(t)=N^{e}[\lambda(t)]$.

It can be seen in Fig. $1(c)$ that the distinguishing features of the transformation-restoring histogram if the synthesis to be transformed has large errors are

(a) a decrease of small values of electron density (segment $B C$ ) and

(b) an increase of large values of electron density (segment $C D$ ).

Segments $A B$ and $D E$ can be present or rot depending on the interrelation of the values $\rho_{\min }^{e}$, $\rho_{\min }^{r}, \rho_{\max }^{e}$ and $\rho_{\max }^{r}$.

We readily see that the central part of the modifying function plot in the HM method is much the same as the plot of modifying functions in the 'classical' DM method. Thus, the modification (16) of the electron-density distribution function with large errors in the modified function can be treated as an approximate transformation restoring the right histogram. In this way we can also think of the DM method as being implicitly based on the specific form of the

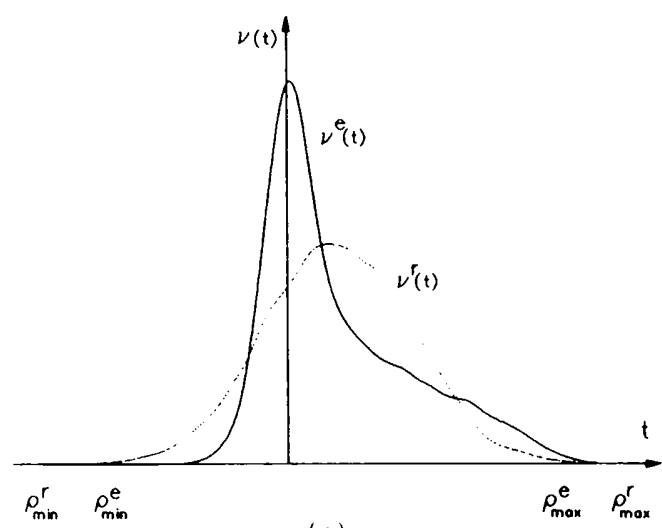

(a)

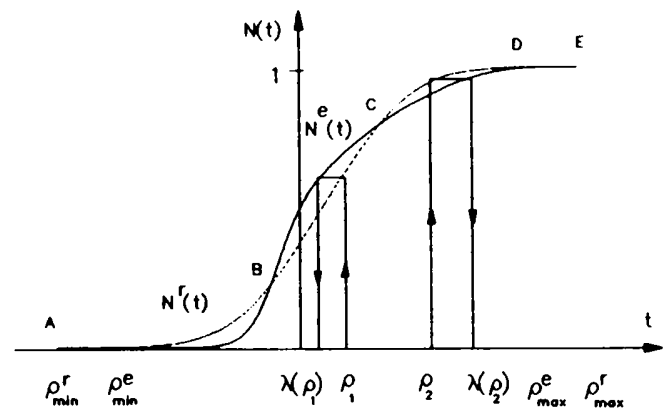

(b)

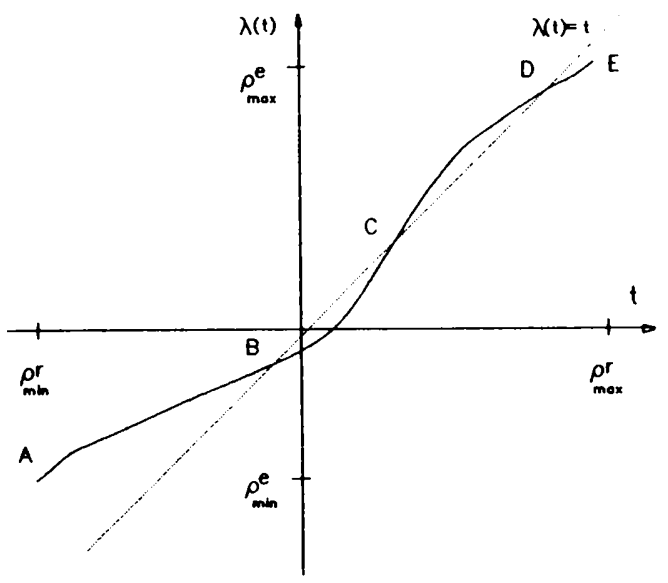

(c)

Fig. 1. (a) Histograms corresponding to syntheses calculated with exact $(-)$ and random $(. . \ldots)$ phases. $(b)$ Cumulative functions for exact- $(-)$ and random-phased $(. \ldots$.$) syntheses.$ (c) The transformation $\lambda(t)$ restoring histogram for randomphased synthesis. 
Table 1. The results of phase refinement (all noncentrosymmetric phases were varied)

\begin{tabular}{|c|c|c|c|c|c|c|c|c|c|}
\hline \multirow[b]{2}{*}{ Method } & \multicolumn{3}{|c|}{$\begin{array}{c}Q_{\Phi} \\
\text { Number of runs }\end{array}$} & \multicolumn{3}{|c|}{$\begin{array}{c}Q_{s} \\
\text { Number of runs }\end{array}$} & \multicolumn{3}{|c|}{$\stackrel{R}{\text { Number of runs }}$} \\
\hline & 0 & 25 & 75 & 0 & 25 & 75 & 0 & 25 & 75 \\
\hline & $72^{\circ}$ & $75^{\circ}$ & - & 0.98 & 0.96 & - & - & - & - \\
\hline GM & $\begin{array}{r}72^{\circ} \\
72^{\circ}\end{array}$ & $71^{\circ}$ & $73^{\circ}$ & $\begin{array}{l}0.98 \\
0.98\end{array}$ & 0.96 & 0.96 & 0.144 & 0.021 & 0.008 \\
\hline
\end{tabular}

Table 2. The results of phase refinement (strongest noncentrosymmetric phases were varied)

\begin{tabular}{cccccccccc} 
& \multicolumn{3}{c}{$\begin{array}{c}Q_{\varphi} \\
\end{array}$} & \multicolumn{4}{c}{ Number of runs } & \multicolumn{3}{c}{$Q_{s}$} & \multicolumn{3}{c}{$R$} \\
Method & 0 & 25 & 75 & 0 & 25 & 75 & 0 & 25 & 75 \\
FRR & $72^{\circ}$ & $53^{\circ}$ & - & 0.98 & 0.86 & - & - & - & - \\
GM & $72^{\circ}$ & $59^{\circ}$ & $58^{\circ}$ & 0.96 & 0.91 & 0.88 & 0.144 & 0.082 & 0.066 \\
DM & $72^{\circ}$ & $55^{\circ}$ & $54^{\circ}$ & 0.96 & 0.88 & 0.88 & 0.600 & 0.741 & 0.742
\end{tabular}

histogram for crystals of biological macromolecules. The difference from the HM method is that its modifying function does not restore the histogram exactly, outlining only the directions in which the synthesis should change.

For small errors in structure-factor phases (and in the respective modified syntheses) the modifying function of the HM method becomes close to the identical transformation $\lambda(t) \equiv t$. In this case, the function (16) distorts the histogram, rather than restores it. So, one can expect the DM method to work less well than the HM method in this case.

\section{Test comparison of the methods}

Several tests were carried out to compare the efficiency of the above methods. The moduli and phases of the structure factors calculated from an atomic model of cytochrome b5 (Mathews, Argos \& Levine, 1972) were used in these tests to check the accuracy of the results. In the phases of noncentrosymmetric reflections calculated from the model (they will in future be referred to as 'exact') we introduced a random error distributed by Von Mises's law

$$
P(\Delta \varphi) \simeq \exp (\lambda \cos \Delta \varphi) .
$$

The parameter $\lambda$ was chosen equal to $0 \cdot 5$, which provided a mean error of bout $70^{\circ}$. The phases of centrosymmetric reflections were taken as exact. Three methods were used to refine the starting-phase values:

(i) FRR minimizing criterion (4) [with the weighting function $w(t)=1$ ];

(ii) GM method;

(iii) DM method with the modifying function (16).

The histogram corresponding to the synthesis calculated with the exact phase values was taken as the standard histogram for FRR and GM methods. In the course of refinement we controlled three characteristics of the current set of phases:

$$
\begin{aligned}
Q_{\varphi} & =\left\langle\left|\varphi_{s}^{\text {ex }}-\varphi_{s}^{c}\right|\right\rangle \\
Q_{s} & =\left\{\int\left[\rho^{\text {ex }}(\mathbf{r})-\rho^{c}(\mathbf{r})\right]^{2} \mathrm{~d} V_{r} / \int\left[\rho^{\text {ex }}(\mathbf{r})\right]^{2} \mathrm{~d} V_{\mathrm{r}}\right\}^{1 / 2} \\
R & =\sum\left|F_{s}^{\text {ex }}-F_{\mathrm{s}}^{m}\right| / \sum\left|F_{s}^{\text {ex }}\right|
\end{aligned}
$$

where $F_{s}^{m}$ are structure-factor moduli for the modified synthesis $\rho^{m}$ in GM and DM methods.

\subsection{Phase refinement of all noncentrosymmetric reflec- tions}

The phases of all noncentrosymmetric reflections were variable parameters in this test. The results of phase refinement by various methods are listed in Table 1. It can be seen from the table that none of the methods was successful.

\subsection{Phase refinement of strong centrosymmetric reflec- tions}

The phases of the strongest noncentrosymmetric reflections were varied in this test (127 reflections from 519). The phases of weak noncentrosymmetric reflections were fixed at wrong values, with a random error introduced. Table 2 shows the results of phase refinement.

One can see from the table that all three methods yielded similar results, although the FRR method showed a more rapid convergence. The failure of the first test can be accounted for by the fact that the histogram may be improved by simultaneous local movements of phases of multiple weak reflections. The prohibition of phase 'synchronization', which allowed the histogram to be improved only on account of strong harmonics, changed qualitatively the course of the refinement.

\section{References}

Harrison, R. W. (1988). J. Appl. Cryst. 21, 949-952.

HOPPE, W. \& GASSMANN, J. (1968). Acta Cryst. B24, 97-107. Lunin, V. Yu. (1985). Acta Cryst. A41, 551-556. 
LUNIN, V. YU. (1986). Use of the Information on Electron Density Distribution in Proteins. Preprint, Pushchino, USSR.

Lunin, V. Yu. (1988). Acta Cryst. A44, 144-150.

Lunin, V. Yu \& Skovoroda, T. P. (1991). Acta Cryst. A47, 45-52.

Lunin, V. Yu., UrzhumtseV, A. G. \& Skovoroda, T. P. (1990). Acta Cryst. A46, 540-544.

Luzzati, V., Mariani, P. \& Delacroix, H. (1988). Makromol. Chem. Macromol. Symp. 15, 1-17.

Mathews, F. S., Argos, P. \& Levine, M. (1972). Cold Spring Harbor Symp. Quant. Biol. 36, 387.
PODJARny, A. D. (1987). In Crystallography in Molecular Biology, edited by D. Moras, J. DRenth, B. Strandberg, D. Suck \& K. Wilson. NATO Adv. Study Inst. Ser. A, 126, 63-79.

Podjarny, A. D. \& Yonath, A. (1977). Acta Cryst. A33, 655-661.

SAYRE, D. (1952). Acta Cryst. 5, 60-65.

SAYRe, D. (1972). Acta Cryst. A28, 210-212.

Simonov, V. I. (1976). In Crystallographic Computing Techniques, edited by F. R. AHMED, K. HuML \& B. SEDLACEK, pp. 138-143. Copenhagen: Munskgaard.

Zhang, K. Y. J. \& Main, P. (1990). Acta Cryst. A46, 41-46.

Acta Cryst. (1991). A47, 243-255

\title{
The Symmetry of Quasiperiodic Systems
}

\author{
BY T. JANSSEN \\ Institute for Theoretical Physics, University of Nijmegen, 6525ED Nijmegen, The Netherlands, \\ and Institut Laue-Langevin, 38042 Grenoble, France
}

(Received 3 August 1990; accepted 11 December 1990)

\begin{abstract}
A discussion is given of the symmetry groups of quasiperiodic systems. This is done in a formalism that uses space groups with dimension larger than three. Three main types are distinguished: modulated crystal phases, incommensurate composite structures and quasicrystals. For these the differences and similarities are discussed and the canonical embedding in higher-dimensional space is given, which requires some generalizations of earlier definitions. The equivalence relation between space groups for quasiperiodic systems is different from that for ordinary space groups, because of the presence of a distinct physical space. Apart from higherdimensional space groups, some quasiperiodic systems have self-similarity properties. Examples are given and the relationship with space-group symmetry is discussed.
\end{abstract}

\section{Introduction}

In the past decades one has found an ever increasing number of structures with perfect order, but without lattice periodicity. These aperiodic 'crystals' are characterized by the fact that their diffraction spots are sharp and may be labelled by a finite number of indices, although this number may be larger than the dimension of the physical space. This means that the basis vectors are linearly dependent, but there is no linear combination with integer coefficients of their vectors which is zero, apart from the trivial case that all coefficients are zero. Such structures have been called quasiperiodic. Actually, lattice periodicity is a special case of quasiperiodicity. Then, the number of

$0108-7673 / 91 / 030243-13 \$ 03.00$ integer indices is equal to the dimension of the space. Quasiperiodic systems are by no means rare (Janssen \& Janner, 1987; Cummins, 1990). Among the minerals the common feldspar may have this property, many dielectrics, like quartz, show it in a certain temperature interval, and some years ago one had artificially made quasicrystals, which also belong to this category.

One can construct mathematical models of structures that are still more general, but nevertheless also perfectly ordered. For example, there are functions that are almost periodic (in the mathematical sense), but not quasiperiodic. (A quasiperiodic function is always almost periodic.) There are chains that can be constructed on a very simple deterministic algorithm, such as the Thue-Morse chain. Also, regular fractals, like the Sierpinski gasket, are perfectly ordered, but not quasiperiodic. All these systems touch on the border of crystallography and seek a generalization of crystallographic concepts. This can most easily be done for quasiperiodic systems to which we shall restrict our considerations here. Because they are in almost every respect similar to crystals, we shall call them aperiodic crystals, although, as the name indicates, generally they lack lattice periodicity.

Because there is no lattice periodicity the usual symmetry description for lattice periodic crystals breaks down. However, as we shall see, we can recover lattice periodicity because quasiperiodic systems are intersections of a lattice periodic system in a higherdimensional space with a hyperplane that represents physical space. The symmetry description then comes down to the description of the higher-dimensional

(C) 1991 International Union of Crystallography 